\title{
An Optimal Retrospective Change Point Detection Policy
}

Running headline: Optimal Change Point Detection Policy

\author{
ALBERT VEXLER \\ Department of Biostatistics, The State University of New York at Buffalo \\ CHENGQING WU \\ Department of Epidemiology and Public Health, Yale University
}

\begin{abstract}
Since the middle of the twentieth century, the problem of making inferences about the point in a surveyed series of observations at which the underlying distribution changes has been extensively addressed in the economics, biostatistics and statistics literature. CUSUM type statistics have commonly been thought to play a central role in non-sequential change point detections. Alternatively, we present and examine an approach based on the Shiryayev-Roberts scheme. We show that retrospective change point detection policies based on Shiryayev-Roberts statistics are non-asymptotically optimal in the context of the most powerful testing.
\end{abstract}

Key words: bayes factors, change point, CUSUM, likelihood, mixture type testing, most powerful testing, optimality, Shiryayev-Roberts

\section{Introduction}

Often, formalized econometric or epidemiological studies correspond to the following problem of testing hypotheses (e.g., Csörgő \& Horváth, 1997). There are $n$ independent data points $X_{1}, X_{2}, \ldots, X_{n}$. The assumption that observations $X_{i}$ are identically distributed (say, $X_{i} \sim$ $\left.f_{0}\left(x ; \theta_{0}\right), 1 \leq i \leq n\right)$ presents the baseline hypothesis $H_{0}$, while the alternative hypothesis $H_{1}$ corresponds to $X_{k} \sim f_{0}\left(x ; \theta_{0}\right), X_{j} \sim f_{1}\left(x ; \theta_{1}\right), k=1, \ldots, \nu-1, j=\nu, \ldots, n$, where $\theta_{0}$ and $\theta_{1}$ are parameters. Thus, we suppose that if a change in the distribution did occur at time $\nu$, then it is unique and the observations after the change all have the same distribution, which differs from the distribution of the observations before the change. Let $P_{\nu}$ and $E_{\nu}$ be the probability measure and expectation conditional on $\nu$ such that, under $P_{\nu}, X_{i} \sim f_{0}\left(x ; \theta_{0}\right) I\{i<\nu\}$ $+f_{1}\left(x ; \theta_{1}\right) I\{i \geq \nu\}, 1 \leq i \leq n$, where $I\{\cdot\}$ is the indicator function and $\nu=1, \ldots, n+1$. 
(The case with $\nu=n+1$ corresponds to $H_{0}$.)

When the parameters $\nu, \theta_{0}$, and $\theta_{1}$ are known, the likelihood methodology suggests the test for $H_{0}$ versus $H_{1}$ based on the ratio

$$
\Lambda_{n}\left(\nu, \theta_{0}, \theta_{1}\right)=\prod_{i=\nu}^{n} \frac{f_{1}\left(X_{i} ; \theta_{1}\right)}{f_{0}\left(X_{i} ; \theta_{0}\right)},
$$

i.e., for a threshold $C$, the event $\left\{\Lambda_{n}\left(\nu, \theta_{0}, \theta_{1}\right)>C\right\}$ declares rejection of $H_{0}$. By virtue of the elementary inequality

$$
(A-B)(I\{A>B\}-\delta) \geq 0, \quad \text { for all } A, B \text {, and } \delta \in[0,1]
$$

with $A=\Lambda_{n}\left(\nu, \theta_{0}, \theta_{1}\right)$ and $B=C$, we have $\left(\Lambda_{n}\left(\nu, \theta_{0}, \theta_{1}\right)-C\right) I\left\{\Lambda_{n}\left(\nu, \theta_{0}, \theta_{1}\right)>C\right\} \geq$ $\left(\Lambda_{n}\left(\nu, \theta_{0}, \theta_{1}\right)-C\right) \delta$. Therefore

$$
E_{n+1}\left(\left(\Lambda_{n}\left(\nu, \theta_{0}, \theta_{1}\right)-C\right) I\left\{\Lambda_{n}\left(\nu, \theta_{0}, \theta_{1}\right)>C\right\}\right) \geq E_{n+1}\left(\left(\Lambda_{n}\left(\nu, \theta_{0}, \theta_{1}\right)-C\right) \delta\right),
$$

where

$$
E_{n+1}\left(\Lambda_{n}\left(\nu, \theta_{0}, \theta_{1}\right) \delta\right)=\int \prod_{i=\nu}^{n} \frac{f_{1}\left(x_{i} ; \theta_{1}\right)}{f_{0}\left(x_{i} ; \theta_{0}\right)} \delta \prod_{i=1}^{n} f_{0}\left(x_{i} ; \theta_{0}\right) \prod_{i=1}^{n} d x_{i}=E_{\nu}(\delta)
$$

for any statistic $\delta$ based on $\left\{X_{1}, \ldots, X_{n}\right\}$. Hence, we obtain

$$
P_{\nu}\left\{\Lambda_{n}\left(\nu, \theta_{0}, \theta_{1}\right)>C\right\}-C P_{n+1}\left\{\Lambda_{n}\left(\nu, \theta_{0}, \theta_{1}\right)>C\right\} \geq P_{\nu}\{\delta=1\}-C P_{n+1}\{\delta=1\},
$$

for any $\delta=0,1$. In this article, we will assume that $\delta=0,1$ is any decision rule based on $\left\{X_{1}, \ldots, X_{n}\right\}$ (for determinacy, say, the event $\{\delta=1\}$ rejects $H_{0}$ ). Thus, supposing that the event $\{\delta=1\}$ rejects $H_{0}$ with type I error $P_{n+1}\{\delta=1\}=\alpha$, we fix the threshold $C: P_{n+1}\left\{\Lambda_{n}\left(\nu, \theta_{0}, \theta_{1}\right)>C\right\}=\alpha$ and conclude that the likelihood ratio statistic (1) provides the most powerful test.

In the case, where the baseline and post-change parameters $\theta_{0}$ and $\theta_{1}$ are known but $\nu$ is unknown, the maximum likelihood estimation of $\nu$ yields the CUSUM statistic

$$
\Lambda_{n}\left(\theta_{0}, \theta_{1}\right)=\max _{1 \leq k \leq n} \Lambda_{n}\left(k, \theta_{0}, \theta_{1}\right), \quad \Lambda_{n}\left(\theta_{0}, \theta_{1}\right)=\frac{f_{1}\left(X_{n} ; \theta_{1}\right)}{f_{0}\left(X_{n} ; \theta_{0}\right)} \max \left(\Lambda_{n-1}\left(\theta_{0}, \theta_{1}\right), 1\right) .
$$

Decision rules based on CUSUM type statistics are well-accepted change point detection schemes (e.g., Page, 1954, 1955; Ploberger \& Kramer, 1992; Sen \& Srivastava, 1975; Pettitt, 1980; Gombay \& Horvath, 1994 as well as Gurevich \& Vexler, 2005). It is widely 
known in change point literature (e.g., Lai, 1995) that likelihood ratio type tests have high power, therefore, evaluation of their significance level is a major issue. Note that $\Lambda_{n}\left(\nu, \theta_{0}, \theta_{1}\right)$ is an $H_{0}$-martingale, whereas its estimator $\Lambda_{n}\left(\theta_{0}, \theta_{1}\right)$ is an $H_{0}$-submartingale (i.e. by the definitions (1) and (4), for $\nu<n$ : $E_{n+1}\left(\Lambda_{n}\left(\nu, \theta_{0}, \theta_{1}\right) \mid X_{1}, \ldots, X_{n-1}\right)=\Lambda_{n-1}\left(\nu, \theta_{0}, \theta_{1}\right)$, but $\left.E_{n+1}\left(\Lambda_{n}\left(\theta_{0}, \theta_{1}\right) \mid X_{1}, \ldots, X_{n-1}\right)=\max \left(\Lambda_{n-1}\left(\theta_{0}, \theta_{1}\right), 1\right) \geq \Lambda_{n-1}\left(\theta_{0}, \theta_{1}\right)\right)$, and moreover, $\Lambda_{n}\left(\theta_{0}, \theta_{1}\right) \geq \Lambda_{n}\left(\nu, \theta_{0}, \theta_{1}\right)$. Thus, the type I error of the test based on the CUSUM statistic can be expected to be raised too high, and hence the idea of a non-asymptotic (as $n$ is fixed) optimality of the retrospective CUSUM test is problematic. (We note, however, that, Moustakides (1986) has shown a non-asymptotical optimality of the sequential change point detection procedure based on the CUSUM statistic.) James et al. (1987) have conjectured that, in the context of the retrospective change point problem, there is no uniformly most powerful test.

Because of the martingale property of the optimal test statistic $\Lambda_{n}\left(\nu, \theta_{0}, \theta_{1}\right)$, it is natural to require that any variation of the detection scheme due to the estimation of $\nu$ should preserve this martingale structure (e.g., Broström, 1997).

In the field of sequential change point detections, professor Pollak and his co-authors have proposed and examined optimal procedures in which a quasi-Bayesian approach to dealing with Shiryayev-Roberts statistics has been applied (e.g., Pollak, 1985). In the context of retrospective detections of changes in complicated models, Vexler (2006) has proposed the Shiryayev-Roberts approach to fix the significance levels of decision rules. The ShiryayevRoberts (SR) statistic has the form of

$$
R_{n}\left(\theta_{0}, \theta_{1}\right)=\sum_{k=1}^{n} \Lambda_{n}\left(k, \theta_{0}, \theta_{1}\right), \quad R_{n}\left(\theta_{0}, \theta_{1}\right)=\frac{f_{1}\left(X_{n} ; \theta_{1}\right)}{f_{0}\left(X_{n} ; \theta_{0}\right)}\left(R_{n-1}\left(\theta_{0}, \theta_{1}\right)+1\right) .
$$

Under $H_{0}$, definition (5) provides $E_{n+1}\left(R_{n} \mid X_{1}, \ldots, X_{n-1}\right)-n=R_{n-1}-(n-1)$. Therefore, the $R_{n}-n$ statistic is an $H_{0}$-martingale. Thus, we can intuitively assume that the retrospective test based on the SR statistic has a significance level that is comparable with the type I error of the likelihood ratio test. Hence, an optimality of the SR test can be shown. Here the expression "optimality" corresponds to the most powerful testing in a variety of contexts. For example, in the next section we demonstrate that the test based on (5) is optimal with respect to the average power (via $\nu=1, \ldots, n$ ) of change point procedures. In this paper, we use the term "optimality" in a manner similar to Lorden's (1967) usage of the term for 
sequential testing.

The main goal of the paper is to present non-asymptotic optimal tests based on the SR statistic for the change point detection. We consider complicated cases of the change point detection where the baseline parameter and post-change parameter are unknown. In the cases where $X \mathrm{~s}$ are stated in terms of a regression model or $X \mathrm{~s}$ are dependent, the proposed approach can be easily extended with support for the optimality of the change point test. A Monte Carlo study confirms that the proposed change point detection policies, in many cases, have power properties that are superior to those of the commonly used CUSUM procedures.

\section{Pre-and-post-change parameters are known}

We begin with the case where parameters $\theta_{0}$ and $\theta_{1}$ are known. (Throughout this paper, $\nu$ is unknown.) Although this case is simple, the situations where baseline and post-change parameters are known, can be associated with real-data problems (e.g., Gurevich \& Vexler, 2005). Here, the analysis is relatively clear, and it has the basic ingredients for more general cases.

The test is given by the following statement: reject $H_{0}$ if and only if

$$
R_{n}\left(\theta_{0}, \theta_{1}\right)>C
$$

where $R_{n}\left(\theta_{0}, \theta_{1}\right)$ is the SR statistic (5) and the threshold $C>0$.

Proposition 1 For the test (6) the following holds:

(i) (6) is the average most powerful change point detection, i.e.

$$
\begin{aligned}
& \frac{1}{n} \sum_{k=1}^{n} P_{k}\left\{R_{n}\left(\theta_{0}, \theta_{1}\right)>C\right\}-\frac{C}{n} P_{n+1}\left\{R_{n}\left(\theta_{0}, \theta_{1}\right)>C\right\} \\
& \geq \frac{1}{n} \sum_{k=1}^{n} P_{k}\left\{\delta \text { rejects } H_{0}\right\}-\frac{C}{n} P_{n+1}\left\{\delta \text { rejects } H_{0}\right\},
\end{aligned}
$$

where $\delta=0,1$ is any decision rule based on $\left\{X_{1}, \ldots, X_{n}\right\}$;

(ii) Under $H_{0}, R_{n}\left(\theta_{0}, \theta_{1}\right) \rightarrow_{n \rightarrow \infty}^{\text {dist }} \gamma$, where $\gamma=\sum_{k=1}^{\infty} \prod_{i=1}^{k} \frac{f_{1}\left(X_{i} ; \theta_{1}\right)}{f_{0}\left(X_{i} ; \theta_{0}\right)}$ is a.s. a finitevalued random variable.

Proof. The expectation under $P_{n+1}$ applied to the inequality (2) with $A=R_{n}\left(\theta_{0}, \theta_{1}\right)$ and 
$B=C$ leads to the inequality

$$
\begin{aligned}
& \sum_{k=1}^{n} E_{n+1}\left(\Lambda_{n}\left(k, \theta_{0}, \theta_{1}\right) I\left\{R_{n}\left(\theta_{0}, \theta_{1}\right)>C\right\}\right)-C P_{n+1}\left\{R_{n}\left(\theta_{0}, \theta_{1}\right)>C\right\} \geq \\
& \sum_{k=1}^{n} E_{n+1}\left(\Lambda_{n}\left(k, \theta_{0}, \theta_{1}\right) I\left\{\delta \text { rejects } H_{0}\right\}\right)-C P_{n+1}\left\{\delta \text { rejects } H_{0}\right\} .
\end{aligned}
$$

Now, by virtue of equation (3), proposition (i) follows.

Lemma 3 of Pollak (1985, p. 210) directly proves statement (ii).

In accordance with Proposition 1, if some test $\delta\left(X_{1}, \ldots, X_{n}\right)$ has type I error $P_{n+1}\{\delta($ $\left.X_{1}, \ldots, X_{n}\right)$ rejects $\left.H_{0}\right\}=\alpha$, then for the fixed threshold $C_{\alpha}: P_{n+1}\left\{R_{n}\left(\theta_{0}, \theta_{1}\right)>C_{\alpha}\right\}=\alpha$, we have

$$
\frac{1}{n} \sum_{k=1}^{n} P_{k}\left\{R_{n}\left(\theta_{0}, \theta_{1}\right)>C_{\alpha}\right\} \geq \frac{1}{n} \sum_{k=1}^{n} P_{k}\left\{\delta \text { rejects } H_{0}\right\} .
$$

Therefore, test (6) has an average power which is greater than that of $\delta\left(X_{1}, \ldots, X_{n}\right)$.

Since the stated problem is parametric, the significance level of (6) can be evaluated by Monte Carlo simulations. Alternatively, Proposition 1 can be applied to evaluate asymptotically (as $n \rightarrow \infty)$ the type I error of the test (6).

Remark. The proof scheme of statement (i) of Proposition 1 provides the next issue. A generalized form of the test (6) can be presented as follows. For $q_{k} \geq 0$, we reject $H_{0}$ if

$$
R_{n}^{q}\left(\theta_{0}, \theta_{1}\right)=\sum_{k=1}^{n} q_{k} \Lambda_{n}\left(k, \theta_{0}, \theta_{1}\right)>C .
$$

In other words, we can take into account a sequence of weights, $\left\{q_{k}\right\}$, that can be considered as a prior distribution of the change point, i.e., $q_{k}=P_{H_{1}}(\nu=k)$. Following the proof of Proposition 1, for all decision rules $\delta\left(X_{1}, \ldots, X_{n}\right)=0,1$ with $P_{n+1}\left\{\delta\left(X_{1}, \ldots, X_{n}\right)\right.$ rejects $\left.H_{0}\right\}=\alpha$, we conclude that

$$
\begin{aligned}
\sum_{k=1}^{n} q_{k} P_{k}\left\{R_{n}^{q}\left(\theta_{0}, \theta_{1}\right)>C_{\alpha}^{q}\right\} \geq & \sum_{k=1}^{n} q_{k} P_{k}\left\{\delta \text { rejects } H_{0}\right\} \\
& C_{\alpha}^{q}: P_{n+1}\left\{R_{n}^{q}\left(\theta_{0}, \theta_{1}\right)>C_{\alpha}^{q}\right\}=\alpha .
\end{aligned}
$$

(The test (7) is the average most powerful change point detection with respect to the weights $q_{k}, k=1, \ldots, n$.) Hence, when the $q_{k}, k=1, \ldots, n$ are not fixed (frequently, in Bayesian cases, $q_{k}, k=1, \ldots, n$ can be pre-specified), these weights can be chosen with respect to a special area of change point occurrence, where the maximum of the test's power is desired corresponding to the interests that belong to a tester. 


\section{Post-change parameter is unknown}

Let $\theta_{0}$ be known, and suppose $\theta_{1}$ is unknown. This case has been dealt with extensively in the literature, and it corresponds to a control problem where the baseline in-control distribution is known and the post-change out-of-control distribution is not. One application of this problem is found in epidemiological studies where it is often assumed that observations have zero expectation prior to a certain unknown change point. (In several situations, when the baseline parameter of an observed sample is unknown, observations can be transformed in order to set a baseline parameter of the transformed sample to a known value, e.g. Brown et al. (1975) as well as Yakir (1998).)

The unknown post-change parameter is usually estimated. To preserve the $H_{0}$-martingale structure of the test statistics, the post-change parameter's estimation has to be adapted with a loss of efficiency (e.g., Lai, 2001 (p. 398), Lorden \& Pollak, 2004 and Gurevich \& Vexler, 2005). (In a general context, Vexler (2008) proposed an approach to obtain $H_{0^{-}}$ martingale type test-statistics with improved power properties.) Alternatively, the literature on sequential change point detection (e.g., Krieger et al., 2003) introduces mixture type tests.

To present the SR statistic when $\theta_{1}$ is not known, we introduce a mixing measure $H(\theta)$ and define

$$
R_{n}\left(\theta_{0}\right)=\sum_{k=1}^{n} \int \Lambda_{n}\left(k, \theta_{0}, \theta\right) d H(\theta)
$$

(Here we can pretend that $\theta_{1} \sim H$, but this assumption is not vital for the execution of the change point detection.) To formulate the next proposition, we point out that the notation

$$
P_{k}\left[L\left(X_{1}, \ldots, X_{n}\right)<u \mid X_{i} \sim\left\{\begin{array}{cc}
f_{0}\left(x ; \theta_{0}\right), & i<k \\
f_{1}(x ; \theta), & i \geq k
\end{array}, i=1, \ldots, n\right]\right.
$$

means a probability distribution function (depending on $k$ and $\theta$ ) of a statistic $L$ based on $X_{i} \sim f_{0}\left(x ; \theta_{0}\right) I\{i<k\}+f_{1}(x ; \theta) I\{i \geq k\}, 1 \leq i \leq n$.

Proposition 2 For the test based on (9) the following hold:

(i) The statistic $R_{n}\left(\theta_{0}\right)-n$ is an $H_{0}$-martingale with respect to the $\sigma$-algebra generated by $X_{1}, \ldots, X_{n}$;

(ii) (9) denotes the average integrated most powerful change point detection with respect 
to $H(\theta)$, i.e.

$$
\begin{aligned}
& \frac{1}{n} \sum_{k=1}^{n} \int P_{k}\left[R_{n}\left(\theta_{0}\right)>C \mid X_{i} \sim\left\{\begin{array}{ll}
f_{0}\left(x ; \theta_{0}\right), & i<k \\
f_{1}(x ; \theta), & i \geq k
\end{array}, i=1, \ldots, n\right] d H(\theta)\right. \\
& -\frac{C}{n} P_{n+1}\left\{R_{n}\left(\theta_{0}\right)>C\right\} \\
& \geq \frac{1}{n} \sum_{k=1}^{n} \int P_{k}\left[\delta \text { rejects } H_{0} \mid X_{i} \sim\left\{\begin{array}{ll}
f_{0}\left(x ; \theta_{0}\right), & i<k \\
f_{1}(x ; \theta), & i \geq k
\end{array}, i=1, \ldots, n\right] d H(\theta)\right. \\
& -\frac{C}{n} P_{n+1}\left\{\delta \text { rejects } H_{0}\right\} \text {, }
\end{aligned}
$$

where $\delta=0,1$ is any decision rule based on $\left\{X_{1}, \ldots, X_{n}\right\}$.

Proof. Since

$$
\begin{aligned}
E_{n+1}\left(R_{n}\left(\theta_{0}\right) \mid X_{1}, \ldots, X_{n-1}\right)-n= & \sum_{k=1}^{n-1} \int \prod_{i=k}^{n-1} \frac{f_{1}\left(X_{i} ; \theta\right)}{f_{0}\left(X_{i} ; \theta_{0}\right)} E_{n+1}\left(\frac{f_{1}\left(X_{n} ; \theta\right)}{f_{0}\left(X_{n} ; \theta_{0}\right)}\right) d H(\theta) \\
& +\int E_{n+1}\left(\frac{f_{1}\left(X_{n} ; \theta\right)}{f_{0}\left(X_{n} ; \theta_{0}\right)}\right) d H(\theta)-n \\
= & \sum_{k=1}^{n-1} \int \prod_{i=k}^{n-1} \frac{f_{1}\left(X_{i} ; \theta\right)}{f_{0}\left(X_{i} ; \theta_{0}\right)}\left(\int \frac{f_{1}(x ; \theta)}{f_{0}\left(x ; \theta_{0}\right)} f_{0}\left(x ; \theta_{0}\right) d x\right) d H(\theta) \\
& +\int\left(\int \frac{f_{1}(x ; \theta)}{f_{0}\left(x ; \theta_{0}\right)} f_{0}\left(x ; \theta_{0}\right) d x\right) d H(\theta)-n=R_{n-1}\left(\theta_{0}\right)-(n-1)
\end{aligned}
$$

the definition (9) preserves the $H_{0}$-martingale property of the test statistic. It is clear that, in a similar manner to the proof scheme of Proposition 1. (i), one can show that, for any test $\delta\left(X_{1}, \ldots, X_{n}\right)$ with significance level $P_{n+1}\left\{\delta\right.$ rejects $\left.H_{0}\right\}=\alpha$,

$$
\begin{aligned}
& \frac{1}{n} \sum_{k=1}^{n} \int P_{k}\left[R_{n}\left(\theta_{0}\right)>C_{\alpha}(H) \mid X_{i} \sim\left\{\begin{array}{ll}
f_{0}\left(x ; \theta_{0}\right), & i<k \\
f_{1}(x ; \theta), & i \geq k
\end{array}, i=1, \ldots, n\right] d H(\theta)\right. \\
& \geq \frac{1}{n} \sum_{k=1}^{n} \int P_{k}\left[\delta \text { rejects } H_{0} \mid X_{i} \sim\left\{\begin{array}{ll}
f_{0}\left(x ; \theta_{0}\right), & i<k \\
f_{1}(x ; \theta), & i \geq k
\end{array}, i=1, \ldots, n\right] d H(\theta),\right.
\end{aligned}
$$

where the threshold $C_{\alpha}(H)$ preserves the significance level $\alpha$ of the test based on (9), i.e. $C_{\alpha}(H)$ is such that $P_{n+1}\left\{R_{n}\left(\theta_{0}\right)>C_{\alpha}(H)\right\}=\alpha$. 


\subsection{Choosing a prior $H(\theta)$}

In the context of sequential change point detection, Krieger et al. (2003) have proposed several forms of a prior $H(\theta)$. The method of Krieger et al. (2003) can be adapted for the problem stated in this paper. For example,

$$
H(\theta)=\Phi\left(\frac{\mu}{\sigma}\right)^{-1}\left(\Phi\left(\frac{\theta-\mu}{\sigma}\right)-\Phi\left(-\frac{\mu}{\sigma}\right)\right)^{+}
$$

if we suspect that the observations after the possible change have a distribution that differs greatly from the distribution of the observations before the change ( $\Phi$ is the standard normal distribution function); a somewhat broader prior is

$$
H(\theta)=\frac{1}{2}\left(\Phi\left(\frac{\theta-\mu}{\sigma}\right)+\Phi\left(\frac{\theta+\mu}{\sigma}\right)\right) .
$$

Note that the parameters $\mu$ and $\sigma>0$ of the distributions $H(\theta)$ can be chosen arbitrarily, e.g., Krieger et al. (2003) have recommended $\mu=0$, and $\sigma=1$ so that (10) and (11) are simplified. Marden (2000) has reviewed the Bayesian approach applied to hypothesis testing. The function $H$ can be defined in accordance with rules mentioned in the literature cited in Section 3 of Marden's paper.

Consider, e.g., for a fixed $\sigma$,

$$
H \in \Theta=\left\{\frac{1}{2}\left(\Phi\left(\frac{\theta-\mu}{\sigma}\right)+\Phi\left(\frac{\theta+\mu}{\sigma}\right)\right), \mu \in\left(\mu_{\text {lower }}, \mu_{\text {upper }}\right)\right\}
$$

(here $\Theta$ is a set of distribution functions). In this case, the definition (9) provides

$$
R_{n}^{a}\left(\theta_{0}\right)=\frac{1}{2} \sum_{k=1}^{n} \int \Lambda_{n}\left(k, \theta_{0}, \theta\right) \frac{\partial\left(\Phi\left(\frac{\theta-a}{\sigma}\right)+\Phi\left(\frac{\theta+a}{\sigma}\right)\right)}{\partial \theta} d \theta .
$$

Let $f_{0}$ and $f_{1}$ be normal densities with standard deviation 1 and means $\theta_{0}=0$ and $\theta_{1}$, respectively. Then the test statistic $R_{n}^{a}\left(\theta_{0}\right)$ has the form of

$$
\begin{aligned}
R_{n}^{a}(0)= & \sum_{k=1}^{n} \frac{1}{2\left(\sigma^{2}(n-k+1)+1\right)^{1 / 2}} \exp \left\{-\frac{a^{2}}{2 \sigma^{2}}\right\} \\
& \times\left[\exp \left\{\frac{\sigma^{2}\left(\sum_{i=k}^{n} X_{i}+a / \sigma^{2}\right)^{2}}{2\left(\sigma^{2}(n-k+1)+1\right)}\right\}+\exp \left\{\frac{\sigma^{2}\left(\sum_{i=k}^{n} X_{i}-a / \sigma^{2}\right)^{2}}{2\left(\sigma^{2}(n-k+1)+1\right)}\right\}\right]
\end{aligned}
$$

(this form is the average integrated most powerful statistic with respect to the distribution function (11), where $\mu=a$ ). Hence, we represent the average integrated power of $R_{n}^{a}(0)$ in 
the form of

$$
\begin{aligned}
& U(a)=\sum_{v=1}^{n} \frac{1}{2} \sum_{j=1}^{2} p_{v j}, \quad p_{v j}=P\left\{\sum_{k=1}^{n} \frac{1}{2\left(\sigma^{2}(n-k+1)+1\right)^{1 / 2}} \exp \left\{-\frac{a^{2}}{2 \sigma^{2}}\right\}\right. \\
& \times\left[\exp \left\{\frac{\sigma^{2}\left(\sum_{i=k}^{n} \varepsilon_{i}+\xi_{j}(n-k+1) I\{k \geq v\}+\xi_{j}(n-v+1) I\{k<v\}+a / \sigma^{2}\right)^{2}}{2\left(\sigma^{2}(n-k+1)+1\right)}\right\}\right. \\
& \left.+\exp \left\{\frac{\sigma^{2}\left(\sum_{i=k}^{n} \varepsilon_{i}+\xi_{j}(n-k+1) I\{k \geq v\}+\xi_{j}(n-v+1) I\{k<v\}-a / \sigma^{2}\right)^{2}}{2\left(\sigma^{2}(n-k+1)+1\right)}\right\}\right] \\
& \left.>C_{\alpha}(a)\right\}, \quad C_{\alpha}(a): P_{n+1}\left\{R_{n}^{a}(0)>C_{\alpha}(a)\right\}=\alpha, \quad \varepsilon_{i} \sim i i d N(0,1), \\
& \xi_{j} \sim N\left(a I\{j=1\}-a I\{j=2\}, \sigma^{2}\right), \quad E \varepsilon_{i} \xi_{j}=0 .
\end{aligned}
$$

To depict the function $U(a)$ from (14), we define $\alpha=0.05$ and $n=20$ for different values of $\sigma$. For each $a$ and $C$, the probability $P_{n+1}\left\{R_{n}^{a}(0)>C\right\}$ was Monte Carlo-estimated via 50000 repetitions of sample $X_{1}, \ldots, X_{n} \sim N(0,1)$. In this way, the approximate values of the roots $C_{0.05}(a)$ were obtained. Similarly, for each $a, v=1, \ldots, n$ and $j=1,2$, the probability $p_{v j}$ by (14) was estimated by Monte Carlo simulation based on 50000 repetitions of the sample $\left\{\varepsilon_{i}, \xi_{j}, i=1, \ldots, n, j=1,2\right\}$.

\section{Figure 1}

Thus, in these cases, if we pretend that $\theta_{1} \sim \Theta$ (were $\Theta$ is given by (12)) then

$$
H(\theta)=\frac{1}{2}\left(\Phi\left(\frac{\theta-\mu}{\sigma}\right)+\Phi\left(\frac{\theta+\mu}{\sigma}\right)\right), \mu=\arg \max _{a=\mu_{\text {lower }}, \mu_{\text {upper }}} U(a)
$$

can be suggested to maximize the average integrated power $U(a)$, when $\mu_{\text {lower }}, \mu_{\text {upper }}$ are fixed corresponding to practical meanings of the testing-statement of problem.

\section{Pre-and-post-change parameters are unknown}

Assume that $\theta_{0}$ and $\theta_{1}$ are unknown. Let $\hat{\theta}_{0}$ denote an estimator of $\theta_{0}$ based on $X_{1}, \ldots, X_{n}$, e.g. $\hat{\theta}_{0}=\arg \max _{a} \prod_{i=1}^{n} f_{0}\left(X_{i} ; a\right)$. Obviously, given an estimation method of $\theta_{0}$, an asymptotic $($ as $n \rightarrow \infty)$ closeness of

$$
R_{n}\left(\hat{\theta}_{0}\right)=\sum_{k=1}^{n} \int \Lambda_{n}\left(k, \hat{\theta}_{0}, \theta\right) d H(\theta)
$$


to the optimal test statistic (9) can be obtained and evaluated. However, in this paper, since we would like to present non-asymptotically optimal procedures, we define

$$
R_{n}=\sum_{k=1}^{n} \int \prod_{i=1}^{k-1} \frac{f_{0}\left(X_{i} ; \bar{\theta}_{0}\right)}{f_{0}\left(X_{i} ; \hat{\theta}_{0}\right)} \Lambda_{n}\left(k, \hat{\theta}_{0}, \bar{\theta}_{1}\right) d H\left(\bar{\theta}_{0}, \bar{\theta}_{1}\right)
$$

where the likelihood ratios $\Lambda_{j}($.$) are denoted by (1) and we pretend that \left(\theta_{0}, \theta_{1}\right) \sim H$. Denoting the estimate of the type I error $P_{n+1}\left\{\delta\right.$ rejects $\left.H_{0}\right\}$ in the form of

$$
\begin{aligned}
& \hat{P}\left\{\delta \text { rejects } H_{0} \mid X_{1}, \ldots, X_{n} \sim f_{0}\left(x ; \hat{\theta}_{0}\right)\right\} \\
& :=\int \prod_{i=1}^{n} f_{0}\left(x_{i} ; \hat{\theta}_{0}\left(x_{1}, \ldots, x_{n}\right)\right) I\left\{\delta\left(x_{1}, \ldots, x_{n}\right) \text { rejects } H_{0}\right\} \prod_{i=1}^{n} d x_{i}=\alpha
\end{aligned}
$$

(here $\hat{P}=P_{n+1}$, if $\hat{\theta}_{0} \equiv \theta_{0}$ ), we formulate the next result.

Proposition 3 Let the estimation method of the unknown baseline parameter be fixed and let $\hat{\theta}_{0}\left(X_{1}, \ldots, X_{n}\right)$ be the estimate of $\theta_{0}$. Then, for all decision rules $\left\{\delta\left(X_{1}, \ldots, X_{n}\right) \in[0,1]\right\}$ with

$$
\hat{P}\left\{\delta \text { rejects } H_{0} \mid X_{1}, \ldots, X_{n} \sim f_{0}\left(x ; \hat{\theta}_{0}\right)\right\}=\alpha \quad(\alpha \text { is pre-specified })
$$

the change point detection based on the statistic (15) is average integrated most powerful with respect to the function $H$.

Proof. Since $\left(R_{n}-C\right)\left(I\left\{R_{n}>C\right\}-\delta\right) \geq 0$, we have

$$
\begin{aligned}
& \sum_{k=1}^{n} \int \prod_{i=1}^{k-1} \frac{f_{0}\left(X_{i} ; \bar{\theta}_{0}\right)}{f_{0}\left(X_{i} ; \theta_{0}\right)} \prod_{i=k}^{n} \frac{f_{1}\left(X_{i} ; \bar{\theta}_{1}\right)}{f_{0}\left(X_{i} ; \theta_{0}\right)} I\left\{R_{n}>C\right\} d H\left(\bar{\theta}_{0}, \bar{\theta}_{1}\right)-C \prod_{i=1}^{n} \frac{f_{0}\left(X_{i} ; \hat{\theta}_{0}\right)}{f_{0}\left(X_{i} ; \theta_{0}\right)} I\left\{R_{n}>C\right\} \\
& \geq \sum_{k=1}^{n} \int \prod_{i=1}^{k-1} \frac{f_{0}\left(X_{i} ; \bar{\theta}_{0}\right)}{f_{0}\left(X_{i} ; \theta_{0}\right)} \prod_{i=k}^{n} \frac{f_{1}\left(X_{i} ; \bar{\theta}_{1}\right)}{f_{0}\left(X_{i} ; \theta_{0}\right)} \delta d H\left(\bar{\theta}_{0}, \bar{\theta}_{1}\right)-C \prod_{i=1}^{n} \frac{f_{0}\left(X_{i} ; \hat{\theta}_{0}\right)}{f_{0}\left(X_{i} ; \theta_{0}\right)} \delta
\end{aligned}
$$

Similar to the proof scheme of the previous propositions, it is clear that deriving $E_{n+1}$ of (17) completes the proof of Proposition 3 .

Thus, (15) is the optimal test-statistic in any set of test-statistics in which the estimation method $\hat{\theta}_{0}$ is applied.

Remark 1. The notation $\hat{P}\left\{\delta\right.$ rejects $\left.H_{0} \mid X_{1}, \ldots, X_{n} \sim f_{0}\left(x ; \hat{\theta}_{0}\right)\right\}$ is not the classical type I error $P_{n+1}\left\{\delta\right.$ rejects $\left.H_{0}\right\}$ ( $\hat{P}$ is the estimate of $P_{n+1}$ given the method $\left.\hat{\theta}_{0}\right)$. When $\hat{\theta}_{0}=$ 
$\arg \max _{a} \prod_{i=1}^{n} f_{0}\left(X_{i} ; a\right)$ (the maximum likelihood estimator), however, any test, which satisfies the conditions of Proposition 3, has the type I error that is in classical control

$$
\begin{gathered}
\sup _{\theta_{0}} P_{n+1}\left\{\delta \text { rejects } H_{0}\right\} \leq \alpha, \text { since } \\
\alpha=\int \prod_{i=1}^{n} f_{0}\left(x_{i} ; \hat{\theta}_{0}\left(x_{1}, \ldots, x_{n}\right)\right) I\left\{\delta\left(x_{1}, \ldots, x_{n}\right) \text { rejects } H_{0}\right\} \prod_{i=1}^{n} d x_{i} \\
\geq \int \prod_{i=1}^{n} f_{0}\left(x_{i} ; \theta_{0}\right) I\left\{\delta\left(x_{1}, \ldots, x_{n}\right) \text { rejects } H_{0}\right\} \prod_{i=1}^{n} d x_{i}, \text { for all } \theta_{0} .
\end{gathered}
$$

Note that, if $\theta_{0}$ is unknown, comparing decision rules from the set

$\left\{\delta: \sup _{\theta_{0}} P_{n+1}\left(\delta\right.\right.$ rejects $\left.\left.H_{0}\right) \leq \alpha\right\}$ (where $\alpha$ is fixed) is a very complex problem. Suppose we have two tests, $(A)$ and $(B)$, for hypothesis $H_{0}^{\prime}$ versus $H_{1}^{\prime}$, given by

$(A)$ : reject $H_{0}^{\prime}$ if statistic $L>C^{L}$; and $(B):$ reject $H_{0}^{\prime}$ if statistic $D>C^{D}$,

where $L, D$ are based on observations $\left\{Z_{i}, i=1, \ldots, m \geq 1\right\}$, and $C^{L}, C^{D}$ are both thresholds. We would like to compare $(A)$ with $(B)$, when the parameters under $H_{0}^{\prime}$ (say, $\eta_{0}$ ) and parameters under $H_{1}^{\prime}$ are unknown. The suggestion of fixing the type I errors of $(A)$ and $(B)$ as $\alpha$ and then contrasting the powers of these tests is problematic. First, in general, we cannot easily choose $C_{\alpha}^{L}, C_{\alpha}^{D}$ such that $\sup _{\eta_{0}} P_{H_{0}^{\prime}}\left\{L>C_{\alpha}^{L}\right\}$ and $\sup _{\eta_{0}} P_{H_{0}^{\prime}}\left\{D>C_{\alpha}^{D}\right\} \leq \alpha$. Since Monte Carlo evaluations of $\sup _{\eta_{0}} P_{H_{0}^{\prime}}$ are usually complex and biased, analytical presentations of $P_{H_{0}^{\prime}}\{L>C\}$ and $P_{H_{0}^{\prime}}\{D>C\}$ are often required to derive $C_{\alpha}^{L}$ and $C_{\alpha}^{D}$. Second, assuming that $C_{\alpha}^{L}$ and $C_{\alpha}^{D}$ are known or evaluated, then comparing $P_{H_{1}^{\prime}}\left\{L>C_{\alpha}^{L}\right\}$ with $P_{H_{1}^{\prime}}\left\{D>C_{\alpha}^{D}\right\}$ is an arduous task $\left(P_{H_{1}^{\prime}}\{\right.$.$\} depends on unknown parameters). Alternatively,$ we suggest fixing $H_{0}^{\prime}$-parameters' estimates (say, $\hat{\eta}_{0}\left(Z_{1}, \ldots, Z_{m}\right)$ ) as well as

$$
\begin{array}{r}
\hat{P}_{H_{0}^{\prime}}\left\{H_{0}^{\prime} \text { is rejected } \mid \hat{\eta}_{0}\right\}:=\int \psi\left(z_{1} ; \hat{\eta}_{0}\left(z_{1}, \ldots, z_{m}\right)\right) \cdots \psi\left(z_{m} ; \hat{\eta}_{0}\left(z_{1}, \ldots, z_{m}\right)\right) \\
\times I\left\{H_{0}^{\prime} \text { is rejected }\right\} d z_{1} \cdots d z_{m}=\alpha
\end{array}
$$

(where $\psi\left(z ; \eta_{0}\right)$ is a density function of $Z$ under $H_{0}^{\prime}$ ), and then evaluating integrated powers of $(A)$ and $(B)$. (When the $\hat{\eta}_{0}$ is equal to true parameters under $H_{0}^{\prime}$, the denoted $\hat{P}_{H_{0}^{\prime}}$ is the significance level.) For example, Proposition 3 provides a method for comparing the test statistic (15) with the CUSUM statistic $\max _{1 \leq k \leq n} \max _{a} \Lambda_{n}\left(k, \hat{\theta}_{0}, a\right)$ under the condition, $\hat{\theta}_{0}$ $=\arg \max _{a} \prod_{i=1}^{n} f_{0}\left(X_{i} ; a\right)$ (see Section 5). Obviously, thresholds $C_{\alpha}^{L}, C_{\alpha}^{D}: \hat{P}_{H_{0}^{\prime}}\left\{L>C_{\alpha}^{L} \mid \hat{\eta}_{0}\right\}$ $=\hat{P}_{H_{0}^{\prime}}\left\{D>C_{\alpha}^{D} \mid \hat{\eta}_{0}\right\}=\alpha$ can be easily obtained by Monte Carlo methods. (We consider calculation $\alpha$-relevant test-thresholds in Section 5 .) 
Remark 2. For the test based on (15), we have

$$
\begin{aligned}
\alpha & =\hat{P}\left\{R_{n}>C \mid X_{1}, \ldots, X_{n} \sim f_{0}\left(x ; \hat{\theta}_{0}\right)\right\}=\int \prod_{i=1}^{n} f_{0}\left(x_{i} ; \hat{\theta}_{0}\left(x_{1}, \ldots, x_{n}\right)\right) \\
& \times I\left\{\prod_{i=1}^{n} f_{0}\left(x_{i} ; \hat{\theta}_{0}\right) \leq \frac{1}{C} \sum_{k=1}^{n} \int \prod_{i=1}^{k-1} f_{0}\left(X_{i} ; \bar{\theta}_{0}\right) \prod_{i=k}^{n} f_{1}\left(x_{i} ; \bar{\theta}_{1}\right) d H\left(\bar{\theta}_{0}, \bar{\theta}_{1}\right)\right\} \prod_{i=1}^{n} d x_{i} \\
& \leq \frac{n}{C} .
\end{aligned}
$$

That is, we have the upper bound (that is independent of different conditions on the distribution of $\left.X_{1}, \ldots, X_{n}\right)$ for $\alpha$. If $\hat{\theta}_{0}$ is the maximum likelihood estimator, then

$$
\sup _{\theta_{0}} P_{n+1}\left\{R_{n}>C\right\} \leq \hat{P}\left\{R_{n}>C \mid X_{1}, \ldots, X_{n} \sim f_{0}\left(x ; \hat{\theta}_{0}\right)\right\} \leq \frac{n}{C} .
$$

Thus, selecting $C=n / \alpha$ determines a test with the level of significance that does not exceed $\alpha$ and ensures a p-value of the test. In accordance with the inequality $\alpha \leq n / C$, theoretically, values of $\alpha$ can be chosen as small as desired. For example, the following test statistics have the same non-asymptotic distribution-free upper bounds for the significance levels:

$$
\begin{array}{rc}
\text { SR: } & \sum_{k=1}^{n} \prod_{i=1}^{k-1} \frac{f_{0}\left(X_{i} ; \hat{\theta}_{0}^{(1, i-1)}\right)}{f_{0}\left(X_{i} ; \hat{\theta}_{0}\right)} \prod_{i=k}^{n} \frac{f_{1}\left(X_{i} ; \hat{\theta}_{1}^{(k, i-1)}\right)}{f_{0}\left(X_{i} ; \hat{\theta}_{0}\right)} ; \\
\text { CUSUM: } & \max _{1 \leq k \leq n} \prod_{i=1}^{k-1} \frac{f_{0}\left(X_{i} ; \hat{\theta}_{0}^{(1, i-1)}\right)}{f_{0}\left(X_{i} ; \hat{\theta}_{0}\right)} \prod_{i=k}^{n} \frac{f_{1}\left(X_{i} ; \hat{\theta}_{1}^{(k, i-1)}\right)}{f_{0}\left(X_{i} ; \hat{\theta}_{0}\right)} ; \\
\text { CUSUM: } & \max _{1 \leq k \leq n} \int \prod_{i=1}^{k-1} \frac{f_{0}\left(X_{i} ; \bar{\theta}_{0}\right)}{f_{0}\left(X_{i} ; \hat{\theta}_{0}\right)} \Lambda_{n}\left(k, \hat{\theta}_{0}, \bar{\theta}_{1}\right) d H\left(\bar{\theta}_{0}, \bar{\theta}_{1}\right),
\end{array}
$$

where $\hat{\theta}^{(r, m)}$ is any estimator (e.g., the MLE) of $\theta$ based on $\left\{X_{r} \ldots, X_{m}\right\}$.

\section{Monte Carlo simulations}

Often in the change point literature, the CUSUM tests have been referred to as the best change point detection policy, particularly in the cases where observations have normal distributions. Thus, we expect good properties of the CUSUM test, when $X_{i}$ has the density function

$$
(2 \pi)^{-0.5} e^{-x^{2} I\{i<\nu\} / 2-\left(x-\theta_{1}\right)^{2} I\{i \geq \nu\} / 2}, i=1, \ldots, n\left(\theta_{0}=0\right) .
$$

In this section, we present simulations to compare the power of the proposed tests with the power of the respective CUSUM detection schemes. To this end, when we estimate the probability of an event $A$, based on $X_{1}, \ldots, X_{n}$, we repeat sampling from (18) 50000 times. The type I error of the considered tests is fixed at $\alpha=0.05$. 
The case when $\theta_{0}$ and $\theta_{1}$ are known. In this case, the proposed test has the form (6), and the CUSUM statistic is $\max _{1 \leq k \leq n} \exp \left\{\theta_{1} \sum_{i=k}^{n} X_{i}-(n-k+1) \theta_{1}^{2} / 2\right\}$. The results of Table 11 are predicted by Proposition [1, i.e. for different $\theta_{1}$ and $n$ :

$$
\frac{1}{n} \sum_{k=1}^{n} P_{k}\left\{R_{n}\left(\theta_{0}, \theta_{1}\right)>C_{0.05}\right\}>\frac{1}{n} \sum_{k=1}^{n} P_{k}\left\{\max _{1 \leq k \leq n} e^{\theta_{1} \sum_{i=k}^{n} X_{i}-(n-k+1) \frac{\theta_{1}^{2}}{2}}>D_{0.05}\right\} .
$$

Table 1

In this simulation study, we observed that the SR test was not only the more powerful on average, but it was also a more powerful detection scheme than CUSUM policy was. As an example we present Figure 2.

\section{Figure 2}

However, we doubt that

$$
P_{\nu}\left\{R_{n}\left(0, \theta_{1}\right)>C_{0.05}\right\} \geq P_{\nu}\left\{\max _{1 \leq k \leq n} e^{\theta_{1} \sum_{i=k}^{n} X_{i}-(n-k+1) \frac{\theta_{1}^{2}}{2}}>D_{0.05}\right\}
$$

for all $\theta_{1}, \nu$, and $n$. To demonstrate the asymptotic result presented in Proposition 1, we define $\gamma=\sum_{k=1}^{50000} \exp \left\{\theta_{1} \sum_{i=1}^{k} X_{i}-k \theta_{1}^{2} / 2\right\}$ and depict the empirical tail probabilities $P_{n+1}\left\{R_{n}>u\right\}$ and $P_{\infty}\{\gamma>u\}$, where $\theta_{1}=0.5$ and $n=20,50,75,100$. (100000 Monte Carlo repetitions of $R_{n}$ and $\gamma$ were conducted.)

\section{Figure 3}

Figure 3 shows that when $n=20$, the tail of the $\gamma$-distribution function cannot be approximated by the distribution function of the SR statistic. However, when $n>50$, the tail probability of SR is close to that of $\gamma$.

The case when $\theta_{1}$ is unknown. Here we analyze the test statistic (9) with $H(\theta)=$ $\Phi\left(\frac{\theta-a}{\sigma}\right)$, i.e.

$$
R_{n}(0)=\sum_{k=1}^{n} \frac{1}{\left(\sigma^{2}(n-k+1)+1\right)^{1 / 2}} \exp \left\{-\frac{a^{2}}{2 \sigma^{2}}+\frac{\sigma^{2}\left(\sum_{i=k}^{n} X_{i}+a / \sigma^{2}\right)^{2}}{2\left(\sigma^{2}(n-k+1)+1\right)}\right\} .
$$

(In this case, $H$ is a commonly used conjugate prior in the context of Bayes Factors methods.) The CUSUM statistic has the form of $\max _{1 \leq k \leq n} \exp \left\{0.5\left(\sum_{i=k}^{n} X_{i}\right)^{2} /(n-k+1)\right\}$. Table 2 
illustrates item (ii) of Proposition 2, where the equation

$$
\begin{aligned}
& \int P_{k}\left[\delta \text { rejects } H_{0} \mid X_{i} \sim\left\{\begin{array}{ll}
f_{0}\left(x ; \theta_{0}\right), & i<k \\
f_{1}(x ; \theta), & i \geq k
\end{array}, i=1, \ldots, n\right] d H(\theta)\right. \\
& =P_{k}\left[\delta \text { rejects } H_{0} \mid X_{i} \sim\left\{\begin{array}{ll}
f_{0}\left(x ; \theta_{0}\right), & i<k \\
f_{1}\left(x ; \theta_{1}\right), & i \geq k
\end{array}, i=1, \ldots, n, \quad \theta_{1} \sim N(a, 1)\right]\right.
\end{aligned}
$$

is applied with $\sigma^{2}=1$.

\section{Table 2}

However, in accordance with Table 3 , the SR test is on average more powerful than the CUSUM test, even when we smooth the unknown $\theta_{1}$ around $\theta_{0}=0$, which is known (i.e. $a=0)$.

\section{Table 3}

Figure 4 shows that when $a \neq \theta_{0}=0$, the SR test is more powerful than the CUSUM test.

\section{Figure 4}

The case, where $\theta_{0}$ and $\theta_{1}$ are unknown. Let the function $d H\left(\bar{\theta}_{0}, \bar{\theta}_{1}\right)$ in (15) have the simple form of $d H\left(\bar{\theta}_{0}, \bar{\theta}_{1}\right)=\varphi\left(\frac{\bar{\theta}_{0}-a_{0}}{\sigma_{0}}\right) \varphi\left(\frac{\bar{\theta}_{1}-a_{1}}{\sigma_{1}}\right) d \bar{\theta}_{0} d \bar{\theta}_{1}$, where $\varphi=d \Phi$. Under $H_{0}$, the maximum likelihood estimator of $\theta_{0}$ is $\hat{\theta}_{0}=\sum_{i=1}^{n} X_{i} / n$. Hence

$$
\begin{aligned}
R_{n}= & \sum_{k=1}^{n} \frac{\exp \left\{-0.5\left(\sum_{i=1}^{n} X_{i}\right)^{2} / n-0.5\left(a_{0}^{2} / \sigma_{0}^{2}+a_{1}^{2} / \sigma_{1}^{2}\right)\right\}}{\left(\sigma_{0}^{2}(k-1)+1\right)^{1 / 2}\left(\sigma_{1}^{2}(n-k+1)+1\right)^{1 / 2}} \\
& \times \exp \left\{\frac{\sigma_{0}^{2}\left(\sum_{i=1}^{k-1} X_{i}+a_{0} / \sigma_{0}^{2}\right)^{2}}{2\left(\sigma_{0}^{2}(k-1)+1\right)}+\frac{\sigma_{1}^{2}\left(\sum_{i=k}^{n} X_{i}+a_{1} / \sigma_{1}^{2}\right)^{2}}{2\left(\sigma_{1}^{2}(n-k+1)+1\right)}\right\}, \text { and } \\
\text { CUSUM }= & \max _{k=1, \ldots, n} \exp \left\{\frac{\left(\sum_{i=k}^{n} X_{i}\right)^{2}}{2(n-k+1)}+\frac{(n-k+1)}{2}\left(\frac{\sum_{i=1}^{n} X_{i}}{n}\right)^{2}\right. \\
& \left.-\frac{\sum_{i=1}^{n} X_{i} \sum_{i=k}^{n} X_{i}}{n}\right\} .
\end{aligned}
$$


Let $\sigma_{0}=\sigma_{1}=1$ and assume that $\theta_{0}=0$. Following Proposition 3 for the SR and CUSUM tests, we fix $\hat{\theta}_{0}\left(X_{1}, \ldots, X_{n}\right)=\sum_{i=1}^{n} X_{i} / n$ and obtain test-thresholds $C_{0.05}$ and $D_{0.05}$ by

$$
\begin{aligned}
\alpha=0.05 & =\int \prod_{i=1}^{n} f_{0}\left(x_{i} ; \hat{\theta}_{0}\left(x_{1}, \ldots, x_{n}\right)=\frac{\sum_{i=1}^{n} x_{i}}{n}\right) I\left\{\delta \text { rejects } H_{0}\right\} \prod_{i=1}^{n} d x_{i} \\
& =\frac{1}{(2 \pi)^{n / 2}} \int e^{\frac{\left(\sum_{i=1}^{n} x_{i}\right)^{2}}{2 n}} I\left\{\delta \text { rejects } H_{0}\right\} e^{-\frac{\sum_{i=1}^{n} x_{i}^{2}}{2}} \prod_{i=1}^{n} d x_{i} \\
& =E_{n+1}\left(e^{\frac{\left(\sum_{i=1}^{n} X_{i}\right)^{2}}{2 n}} I\left\{\delta\left(X_{1}, \ldots, X_{n}\right) \text { rejects } H_{0}\right\}\right) .
\end{aligned}
$$

(We numerically evaluated the corresponding classical type I error of the SR and CUSUM procedures: $P_{n+1}\left\{\right.$ SR rejects $\left.H_{0}\right\} \simeq P_{n+1}\left\{\right.$ CUSUM rejects $\left.H_{0}\right\} \simeq 0.02$.)

Similar to Table 2 in the previous paragraph, Table 4 relates to Proposition 3.

\section{Table 4}

In the considered cases, Table 5] concludes that regardless of a prior distribution of the unknown parameters, the SR test is on average more powerful than the CUSUM test. For example, when we set $n=100, \theta_{1}=0.7$, and $d H\left(\bar{\theta}_{0}, \bar{\theta}_{1}\right)=\varphi\left(\bar{\theta}_{0}\right) \varphi\left(\bar{\theta}_{1}-1\right) d \bar{\theta}_{0} d \bar{\theta}_{1}$, the average power of the SR test is 0.5048 whereas the average power of the CUSUM test is 0.2389 .

\section{Table 5}

On the level of the $P_{k}$-regime, the situations of $\left\{a_{0}=0, a_{1}=0\right\},\left\{a_{0}=0, a_{1}=0.5\right\},\left\{a_{0}=\right.$ $\left.0.5, a_{1}=0\right\}$, and $\left\{a_{0}=0, a_{1}=1\right\}$ are displayed in Figure 5 .

\section{Figure 5}

Note that, certainly, the case $\left\{a_{0}=0, a_{1}=0\right\}$ (both the pre-and-post change parameters are expected to be around 0$)$ is strange and the case $\left\{a_{0}=0.5, a_{1}=0\right\}$ corresponds to the misunderstanding of a physical process presented by an analyzed data set. (In this situation, (10) or (11) type functions for $H$ can be recommended because such functions regard no information about $\theta_{0}<\theta_{1}$.) Even in these cases, however, in most situations, the SR procedure is still more powerful than the CUSUM procedure.

Thus, the results of the Monte Carlo simulations experimentally confirm the existence of a practical meaning of the proposed tests. Although we did not utilize any technique to choose priors for the unknown parameters, the Monte Carlo study showed that the proposed change point detections are somewhat better than the well-known CUSUM-procedures. 


\section{Remarks}

1. Assume that $\theta_{0}$ and $\theta_{1}$ are multidimensional parameters. In this case, the proposed change point detection method does not need to be changed. When the dimension of the vector of unknown parameters is close to the number of observations, we suspect that the proposed testing is very reasonable. Note that the proposed method can be easily adapted to regression models. In several situations, biostatisticians deal with "wide and short" data, where the number of explanatory variables is close to the number of observations. In these situations, applications of tests based on the estimation of unknown parameters are problematic.

2. The proposed method can be modified if $X_{i}, i \geq 1$ are non-independent random variables. For example, in this case, we denote

$$
R_{n}\left(\theta_{0}, \theta_{1}\right)=\sum_{k=1}^{n} \prod_{i=k}^{n} \frac{f_{1}\left(X_{i} \mid X_{1}, \ldots, X_{i-1} ; \theta_{1}\right)}{f_{0}\left(X_{i} \mid X_{1}, \ldots, X_{i-1} ; \theta_{0}\right)} .
$$

Obviously, the modified tests have the optimal properties that are mentioned in the propositions of this paper.

3. Consider the problem of change-points with epidemic alternatives (e.g., Vexler, 2006, 2008), i.e., $X_{i}$ has density $f_{0} I\left\{i<\nu_{1}\right\}+f_{1} I\left\{\nu_{1} \leq i<\nu_{2}\right\}+f_{0} I\left\{i \geq \nu_{2}\right\}$, where $i=1, \ldots, n$ and $\nu_{1}<\nu_{2}$ are unknown. In order to test for $\nu_{1}>n$ versus $\nu_{1} \leq n$, the proposed method can be easily extended, i.e.

$$
R_{n}\left(\theta_{0}, \theta_{1}\right)=\sum_{k=1}^{n} \sum_{r=1}^{k} \prod_{i=r}^{k} \frac{f_{1}\left(X_{i} ; \theta_{1}\right)}{f_{0}\left(X_{i} ; \theta_{0}\right)} .
$$

4. In accordance with Remark of Section 2, Propositions 2 and 3 , weights $\left\{q_{k}, k=1, \ldots, n\right\}$ and/or prior $H$ can be chosen with respect to a special area of parameters $\nu, \theta_{0}, \theta_{1}$, under the alternative hypothesis, where the maximum of the test's power is desired. Thus, the proposed change point tests provide maximum integrated power in the area that can correspond to the tester's interests.

\section{Acknowledgements}

This research was supported by the Intramural Research Program of the National Institute 
of Child Health and Human Development, National Institutes of Health. The opinions expressed are those of the authors and not necessarily of the National Institutes of Health. The authors are indebted to Professors Kai Fun Yu and Aiyi Liu for many helpful discussions and comments. We are grateful to the editor, associate editor, and two referees for their helpful comments that clearly improved this paper.

\section{References}

Broström, G. (1997). A martingale approach to the changepoint problem J. Amer. Statist. Assoc. 92, 1177-1183.

Brown, R. L., Durbin, J. \& Evans, J. M. (1975). Techniques for testing the constancy of regression relationships over time (with discussion). J. Roy. Statist. Soc. Ser. B 37, 149-192.

Csörgö, M. \& Horváth, L. (1997). Limit theorems in change-point analysis. Wiley, New York.

Gombay, E. \& Horvath, L. (1994). An application of the maximum likelihood test to the change-point problem. Stochastic Process. Appl. 50, 161-171.

Gurevich, G. \& Vexler, A. (2005). Change point problems in the model of logistic regression. J. Statist. Plann. Inference 131, 313-331.

James, B., James K. L. \& Siegmund D. (1987). Tests for a change-point. Biometrika 74, 71-83.

Krieger, A. M., Pollak, M. \& Yakir, B. (2003). Surveillance of a simple linear regression. J. Amer. Statist. Assoc. 98, 456-469.

Lai, T.L. (1995). Sequential changepoint detection in quality control and dynamical systems. J. Roy. Statist. Soc. Ser. B. 57 4, 613-658.

Lai, T.L. (2001). Sequential analysis: some classical problems and new challenges. Statist. Sinica 11, 303-408. 
Lorden, G. (1967). Integrated risk of asymptotically bayes sequential tests. Ann. Math. Statist. 38, 1399-1422.

Lorden, G. \& Pollak, M. (2004). Non-anticipating estimation applied to sequential analysis and changepoint detection. Ann. Statist. 33, 1422-1454.

Marden, J. I. (2000). Hypothesis testing: from p values to Bayes factors. J. Amer. Statist. Assoc. 95, 1316-1320.

Moustakides G. V. (1986). Optimal stopping times for detecting changes in distributions. Ann. Statist. 14, 1379-1387.

Page, E. S. (1954). Continuous inspectation schemes. Biometrika 41, 100-114.

Page, E. S. (1955). A test for a change in a parameter occurring at an unknown Point. Biometrika 42, 523-527.

Pettitt, A. N. (1980). A simple cumulative sum type statistic for the change-point with zero-one observations. Biometrika 67, 79-84.

Ploberger, W. \& Kramer, W. (1992). The Cusum Test with Ols Residuals. Econometrica 60, 271-285.

Pollak, M. (1985). Optimal detection of a change in distribution. Ann. Statist. 13, 206227.

Sen, A. \& Srivastava, M. S. (1975). On tests for detecting change in mean. Ann. Statist. 1, 98-108.

Vexler, A. (2006). Guaranteed testing for epidemic changes of a linear regression model. J. Statist. Plann. Inference 136, 3101-3120.

Vexler, A. (2008). Martingale type statistics applied to change points detection. Comm. Statist. Theory Methods 37, 1207-1224.

Yakir, B. (1998). On the average run length to false alarm in surveillance problems which possess an invariance structure. Ann. Statist. 26, 1198-1214. 
Albert Vexler, Department of Biostatistics, The State University of New York, Buffalo, NY, 14214, USA.

E-mail: avexler@buffalo.edu 


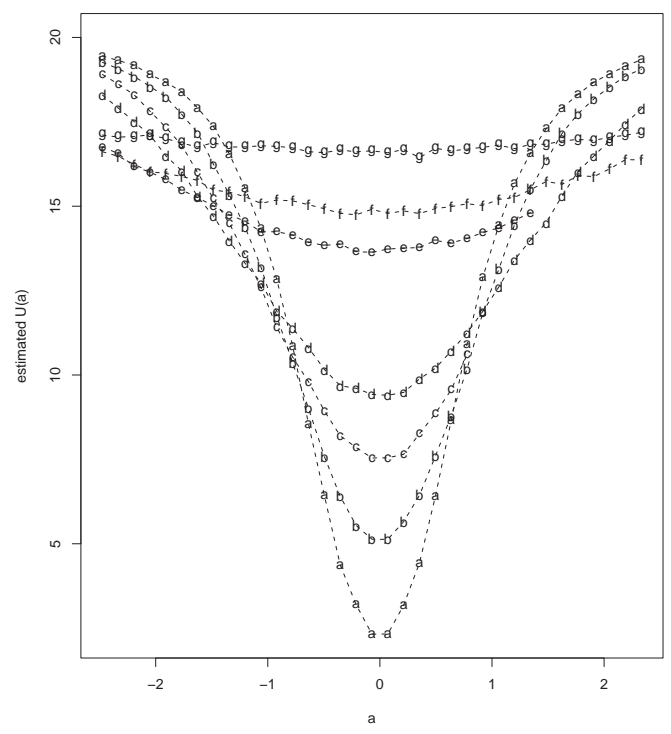

Figure 1: The Monte Carlo estimator of the function $U(a)$ from (14). The curves -a-, -b-, -c-, -d-, -e-, -f- and -g- correspond to $\sigma=0.25,0.5,0.75,1,2,2.5$ and 4 , respectively.

Table 1: The Monte Carlo estimators of the average powers $\sum_{k=1}^{n} P_{k}\left\{\delta\right.$ rejects $\left.H_{0}\right\} / n$, where the decision rules $\delta$ are the SR and CUSUM tests.

\begin{tabular}{|c|ccccccc|}
\hline & & $\theta_{1}=0.1$ & $\theta_{1}=0.2$ & $\theta_{1}=0.3$ & $\theta_{1}=0.5$ & $\theta_{1}=0.7$ & $\theta_{1}=1$ \\
\hline$n=10$ & SR & 0.0738 & 0.1076 & 0.1516 & 0.2708 & 0.4132 & 0.6217 \\
& CUSUM & 0.0732 & 0.1067 & 0.1457 & 0.2651 & 0.4087 & 0.6123 \\
\hline$n=20$ & SR & 0.0892 & 0.1551 & 0.2543 & 0.4292 & 0.5999 & 0.7898 \\
& CUSUM & 0.0842 & 0.1356 & 0.2074 & 0.3953 & 0.5858 & 0.7758 \\
\hline$n=100$ & SR & 0.1549 & 0.3503 & 0.5639 & 0.8230 & 0.9165 & 0.9591 \\
& CUSUM & 0.1488 & 0.3343 & 0.5452 & 0.8042 & 0.9025 & 0.9544 \\
\hline
\end{tabular}

Table 2: The Monte Carlo estimators of the average powers $\sum_{k=1}^{n} P_{k}\left\{\delta\right.$ rejects $\left.H_{0}\right\} / n$, where the decision rules $\delta$ are the SR and CUSUM tests and the unknown post-change parameter $\theta_{1}$ is generated from normal distributions.

\begin{tabular}{|c|cccc|}
\hline \multirow{2}{*}{$n=20$} & $\theta_{1}$ & $\sim N(0,1)$ & $\sim N(0.5,1)$ & $\sim N(1,1)$ \\
\cline { 2 - 5 } & SR & 0.4883 & 0.5371 & 0.6352 \\
\hline \multirow{2}{*}{$n=100$} & CUSUM & 0.4625 & 0.5027 & 0.6081 \\
\cline { 2 - 5 } & SR & 0.6997 & 0.7491 & 0.8332 \\
\cline { 2 - 5 } & CUSUM & 0.6879 & 0.7178 & 0.7923 \\
\hline
\end{tabular}




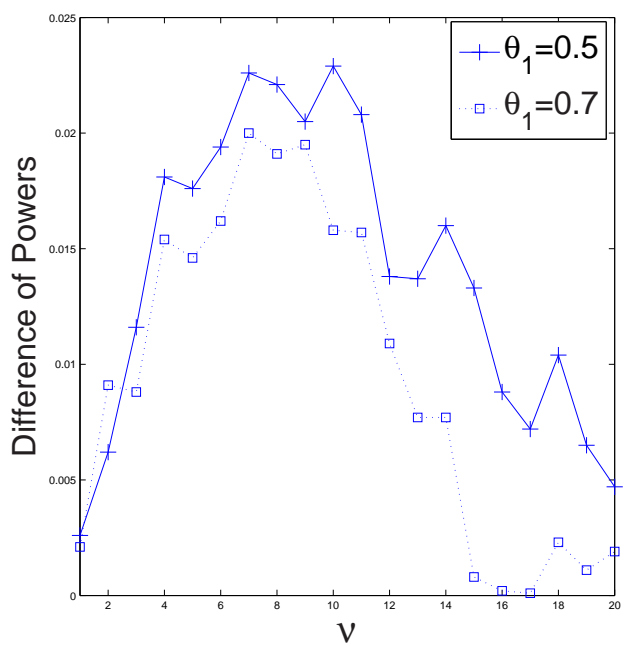

Figure 2: The Monte Carlo estimators of $P_{\nu}\left\{\mathrm{SR}\right.$ rejects $\left.H_{0}\right\}-P_{\nu}\left\{\right.$ CUSUM rejects $\left.H_{0}\right\}$, where $P_{n+1}\left\{\right.$ SR rejects $\left.H_{0}\right\}=P_{n+1}\left\{\right.$ CUSUM rejects $\left.H_{0}\right\}=0.05$ and $n=20$.

Table 3: The Monte Carlo estimators of the average powers of the SR and CUSUM tests, for different values of the post-change parameter $\theta_{1}$.

\begin{tabular}{|c|c|ccc|ccc|}
\hline \multicolumn{2}{|c|}{$\sigma^{2}=1$} & \multicolumn{3}{c|}{$n=20$} & \multicolumn{3}{c|}{$n=100$} \\
\multicolumn{2}{|c|}{} & $a=0.0$ & $a=0.5$ & $a=1.0$ & $a=0.0$ & $a=0.5$ & $a=1.0$ \\
\hline$\theta_{1}=0.1$ & CUSUM & & 0.0598 & & & 0.0813 & \\
& SR & 0.0610 & 0.0699 & 0.0774 & 0.1009 & 0.1059 & 0.1233 \\
\hline$\theta_{1}=0.5$ & CUSUM & & 0.2751 & & & 0.7127 & \\
& SR & 0.3071 & 0.3539 & 0.3741 & 0.7649 & 0.7798 & 0.8091 \\
\hline$\theta_{1}=0.7$ & CUSUM & & 0.4707 & & & 0.8526 & \\
& SR & 0.4835 & 0.5509 & 0.5699 & 0.8696 & 0.8874 & 0.8959 \\
\hline
\end{tabular}

Table 4: The Monte Carlo estimators of the average powers $\sum_{k=1}^{n} P_{k}\left\{\delta\right.$ rejects $\left.H_{0}\right\} / n$, where the zdecision rules $\delta$ are the SR and CUSUM tests and the unknown pre-post-change parameters $\theta_{0}, \theta_{1}$ are generated from normal distributions.

\begin{tabular}{|c|c|c|c|c|c|}
\hline \multicolumn{2}{|c|}{$\theta_{j} \sim N\left(a_{j}, 1\right), j=0,1$} & $a_{0}=0, a_{1}=0$ & $a_{0}=0, a_{1}=.5$ & $a_{0}=.5, a_{1}=.0$ & $a_{0}=0, a_{1}=1$ \\
\hline$n=20$ & RS & 0.2824 & 0.3191 & 0.3129 & 0.3697 \\
& CUSUM & 0.2173 & 0.2366 & 0.2359 & 0.2902 \\
\hline$n=100$ & RS & 0.5534 & 0.5872 & 0.5854 & 0.6489 \\
& CUSUM & 0.4524 & 0.4731 & 0.4724 & 0.5292 \\
\hline
\end{tabular}



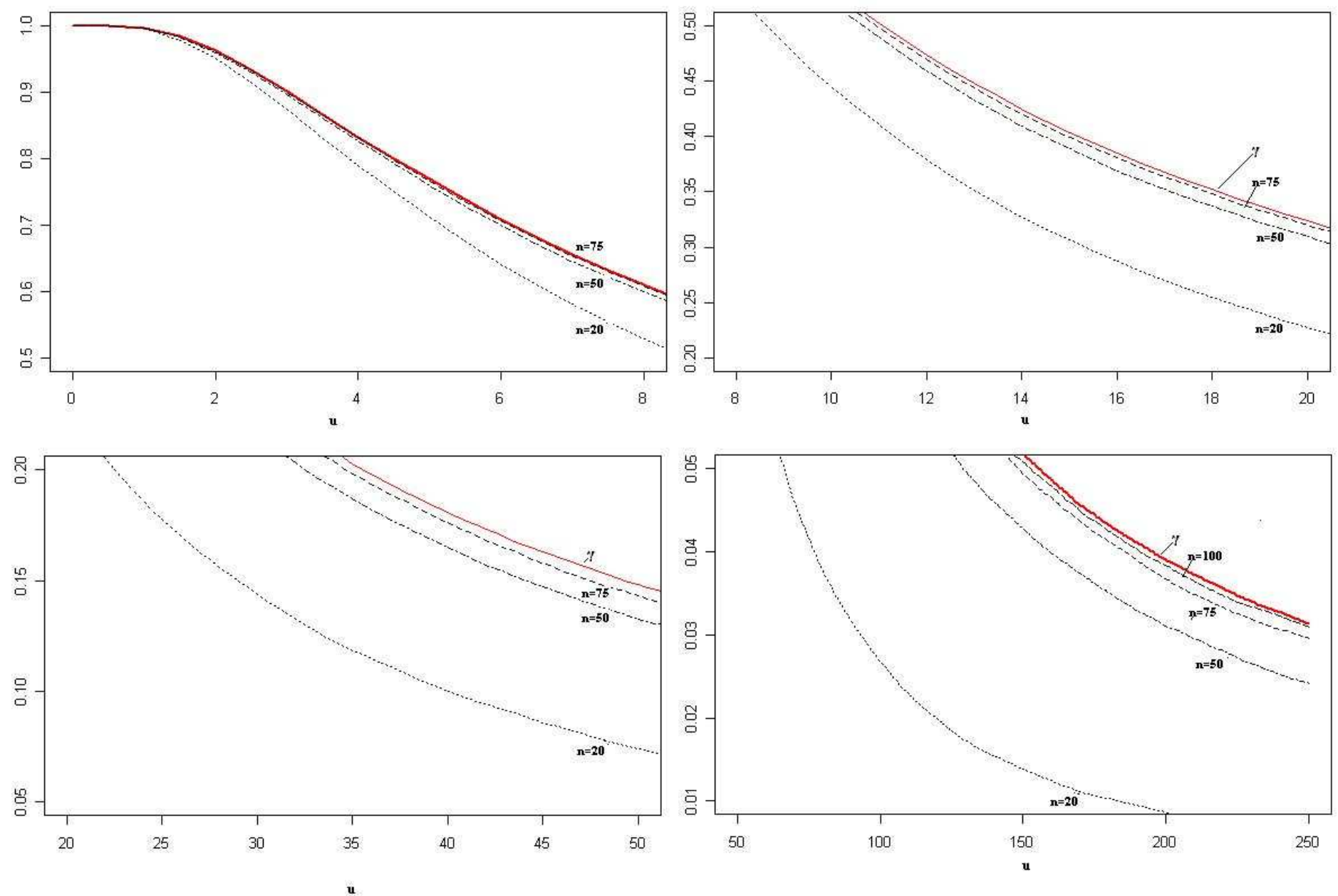

Figure 3: The Monte Carlo probabilities $P_{n+1}\left\{R_{n}>u\right\}$ and $P_{\infty}\{\gamma>u\}$ are depicted by the line types that are specified as "dotted", "dotdash", "dashed" and "longdash" corresponding to $n=20,50,75$ and $n=100$, respectively. Here $\gamma$ is denoted in Proposition 1 and $\theta_{1}=0.5$.

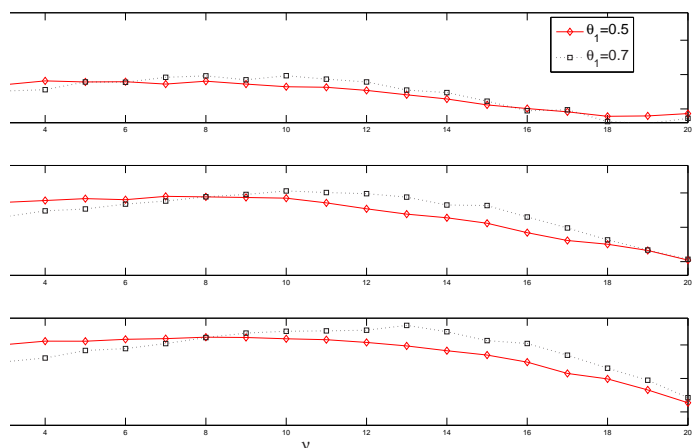

Figure 4: The Monte Carlo estimators of $P_{\nu}\left\{\mathrm{SR}\right.$ rejects $\left.H_{0}\right\}-P_{\nu}\left\{\mathrm{CUSUM}\right.$ rejects $\left.H_{0}\right\}$, where $P_{n+1}\left\{\right.$ SR rejects $\left.H_{0}\right\}=P_{n+1}\left\{\right.$ CUSUM rejects $\left.H_{0}\right\}=0.05$ and $n=20$. 


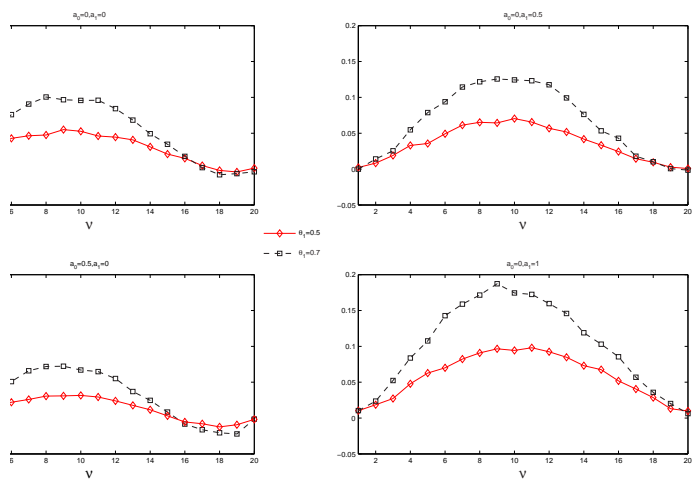

Figure 5: The Monte Carlo estimators of $P_{\nu}\left\{\mathrm{SR}\right.$ rejects $\left.H_{0}\right\}-P_{\nu}\left\{\right.$ CUSUM rejects $\left.H_{0}\right\}$, where $\hat{P}_{n+1}\left\{\right.$ SR rejects $\left.H_{0}\right\}=\hat{P}_{n+1}\left\{\right.$ CUSUM rejects $\left.H_{0}\right\}=0.05$ and $n=20$.

Table 5: The Monte Carlo estimators of the average powers of the SR and CUSUM tests, for different values of the post-change parameter $\theta_{1}$ (the pre-change parameter $\theta_{0}$ ).

\begin{tabular}{|c|c|c|c|c|c|c|}
\hline \multicolumn{3}{|c|}{$\sigma_{0}=\sigma_{1}=1$} & $a_{0}=0, a_{1}=0$ & $a_{0}=0, a_{1}=.5$ & $a_{0}=.5, a_{1}=.0$ & $a_{0}=0, a_{1}=1$ \\
\hline \multirow{6}{*}{$n=20$} & \multirow{2}{*}{$\theta_{1}=0.1$} & $\mathrm{RS}$ & 0.0220 & 0.0200 & 0.0186 & 0.0254 \\
\hline & & CUSUM & \multicolumn{4}{|c|}{0.0153} \\
\hline & \multirow[t]{2}{*}{$\theta_{1}=0.5$} & $\mathrm{RS}$ & 0.0538 & 0.0632 & 0.0401 & 0.0864 \\
\hline & & CUSUM & \multicolumn{4}{|c|}{0.0331} \\
\hline & \multirow[t]{2}{*}{$\theta_{1}=0.7$} & $\mathrm{RS}$ & 0.0905 & 0.1104 & 0.0766 & 0.1460 \\
\hline & & CUSUM & \multicolumn{4}{|c|}{0.0558} \\
\hline \multirow[t]{6}{*}{$n=100$} & \multirow[t]{2}{*}{$\theta_{1}=0.1$} & RS & 0.0169 & 0.0189 & 0.020 & 0.0239 \\
\hline & & CUSUM & \multicolumn{4}{|c|}{0.0148} \\
\hline & \multirow[t]{2}{*}{$\theta_{1}=0.5$} & $\mathrm{RS}$ & 0.1999 & 0.2289 & 0.2264 & 0.2451 \\
\hline & & CUSUM & \multicolumn{4}{|c|}{0.0958} \\
\hline & \multirow[t]{2}{*}{$\theta_{1}=0.7$} & RS & 0.4245 & 0.4689 & 0.4519 & 0.5048 \\
\hline & & CUSUM & \multicolumn{4}{|c|}{0.2389} \\
\hline
\end{tabular}

